\title{
Active Fault Tolerance Control Based on Consistent Matrix for Multimotor Synchronous System
}

\author{
Haijie Mao $\mathbb{D}^{1,2,3}$ Wei Li $\mathbb{D}^{1,2,3}$ Xiaolin Feng $\mathbb{D}^{1,2,3}$ and Haochuan Zhang ${ }^{1}$ \\ ${ }^{1}$ College of Electrical and Information Engineering, Lanzhou University of Technology, Lanzhou 730050, China \\ ${ }^{2}$ Key Laboratory of Gansu Advanced Control for Industrial Processes, Lanzhou 730050, China \\ ${ }^{3}$ National Demonstration Center for Experimental Electrical and Control Engineering Education, \\ Lanzhou University of Technology, Lanzhou 730050, China \\ Correspondence should be addressed to Xiaolin Feng; splendor2003@126.com
}

Received 9 November 2019; Revised 18 January 2020; Accepted 5 February 2020; Published 21 April 2020

Academic Editor: Xianming Zhang

Copyright (C) 2020 Haijie Mao et al. This is an open access article distributed under the Creative Commons Attribution License, which permits unrestricted use, distribution, and reproduction in any medium, provided the original work is properly cited.

\begin{abstract}
This paper presents an active fault-tolerant method to mitigate sensor failures in multimotor synchronous control. First, inspired by the construction of the coupling matrix in complex network synchronous output, a consistent matrix is designed based on structural redundancy in synchronous control. This consistent matrix has two advantages: one is that it can reflect different sensor output similarities and the other one is that it can detect, locate, and estimate the sensor fault. Then, the fault information is integrated into the design of tolerance control with an improved mean feedback mechanism. The proposed method is suitable for both single and multiple fault situations, and its effectiveness is finally verified by both MATLAB simulation and the ABB semiphysical experimental platform.
\end{abstract}

\section{Introduction}

Due to great load drive capabilities and more flexible motion modes, multimotor synchronous driven systems have been widely applied in numerous industry domains including robotics, paper making, and belt conveyors [1-4]. The objective of synchronous control is to ensure synchronization of speed or displacement between different motors under different loads or disturbances [5-7]. In multimotor synchronous control systems, the sensors used to measure speed or position are easily malfunctioned due to ageing, collision, or electromagnetic interference. Once a fault occurs, the synchronization control performance will be seriously affected and disastrous consequences may happen. Therefore, identifying such faults and taking effective measures in time is of great significance for ensuring system safety, reliability, and product quality $[5,6]$.

The development of fault detection and diagnosis (FDD) and fault-tolerant control (FTC) based on analytical redundancy provides strong support for ensuring the security of the system. Many researchers have studied FTC for sensor faults in motion control systems for decades [8-15]. They can be roughly divided into two categories, namely, mathematical model-based and data-driven-based [9]. The core of the mathematical model method is the design of various types of observers. For example, Mao et al. [10] adopted adaptive disturbance rejection control (ADRC) and an extended state observer (ESO) to estimate the speed in a current loop, which realized FTC for a speed sensor fault and improved the multimotor synchronization accuracy. Najafabadi et al. [11] solved the problem of diagnosing and isolating three sensor faults for current, voltage, and speed in induction motors through designing an adaptive current observer for rotor resistance estimation. For a speed sensor failure in an induction motor, Marino et al. [12] designed an adaptive observer to detect sensor faults online, and fault tolerance was realized based on indirect magnetic field orientation control. In recent years, aiming at the difficulty of modelling, the data-driven method has gradually become a new research hot spot, which has received extensive attention [13-15]. Based on the large amount of online and offline monitoring data existing in the system, this method 
can characterize the normal and fault modes in the system based on that useful information hidden in data using data mining and processing technologies. Consequently, it has been recognized as a practical diagnostic technology. As an application, by collecting line voltage and performing fast Fourier transform, a data-driven method using a two-layer Bayesian network was proposed in [13] to obtain fault characteristics. As a result, fault diagnosis of an inverter in PMSM was realized.

Recalling the existing results in the literature, most results are directed to single-motor drives. Although some of them are also applicable to multimotor synchronous driving modes, they still have some limitations since the presented algorithms are complicated and difficult to implement. To overcome such limitations, in this paper, through exploring mathematical model-based and data-driven-based methods' characters and shortages, we devise a combination method based on a complex network.

The complex network model has been widely used in many fields, such as power grids and aerospace [16-19]. A typical complex network system consists of a number of subsystems that are usually coupled with each other. Typically, a multimotor synchronous control system is such a complex network. The consistency study of complex networks, that is, complex network synchronization control, is an important research topic in the field of complex networks.

Redundancy is the basis of fault diagnosis and fault tolerance. In this paper, inspired by the construction of a coupling matrix in complex network systems, a consistent matrix is designed to characterize the similarities of output data of different sensors, using the structure and information redundancy in multimotor synchronous control systems. Based on online analysis and judgment of matrix elements and eigenvalues, the detection, location, and estimation for faulty sensors are realized. As a result, a novel improved mean feedback strategy is presented using structural redundancy and fault information to achieve fault tolerance. It is shown through simulation and experiment that system security and reliability are greatly improved.

\section{Deviation-Coupled Synchronous Control Structure}

The research on multimotor synchronous control strategies mainly includes two aspects, synchronous control structure and synchronous control algorithm. In terms of the control structure, there are mainly serial master-slave control [20], virtual spindle control [21], cross-coupling control [22, 23], and deviation-coupling control [24]. Among them, deviation coupling adopts the compensation control strategy and the comprehensive effect is more positive than others in aspects of starting characteristics, disturbance suppression ability, applicable range, and convenience of engineering realization. Accordingly, its practical applications have been found in a wider range of fields. In terms of control algorithms, which are mainly for load uncertainty and unknown interference, various robust control algorithms are devised [10, 24-27], such as sliding mode control, internal model control, and active disturbance rejection control. Since this paper focuses on fault diagnosis and tolerance in multimotor synchronous control systems, the deviation-coupled synchronous control structure in [10] is adopted. The control principle is shown in Figure 1, where ADRC is a kind of controller and S.C. is the synchronous compensator or controller, such as PI or PID.

\section{Sensor Fault FDD and FTC Design Based on Complex Network Consistent Matrix}

\subsection{Establishment of Complex Dynamical Network Consistent Matrix}

3.1.1. Complex Network Coupling Matrix. Based on graph theory, a complex network system with identical dynamic systems as nodes that satisfies the dissipation conditions can be described as follows [28]:

$$
\dot{x}_{i}=f\left(x_{i}(t)\right)+\sigma \sum_{j=1}^{N} a_{\mathrm{ij}} H\left(x_{j}(t)\right),
$$

where $x_{i}=\left(x_{i 1}, x_{i 2}, \ldots, x_{i N}\right)^{T} \in R^{n}$ is the state of node $i$, $i=1,2, \ldots, N ; f \in C\left[R^{n}, R^{n}\right]$ is a known function (often a nonlinear function); $\sigma>0$ is the coupling strength of the network; $A=\left(a_{i j}\right)_{N \times N}$ is the coupling matrix of the network, which satisfies $\sum_{j} a_{i j}=0, i=1,2, \ldots, N$; and $H \in C\left[R^{n}, R^{n}\right]$, which is a coupling function.

The coupling matrix $A$ can be used to describe an undirected topology. If there is a connection between node $i$ and node $j$, then $a_{i j}=a_{j i}=k, k>0$, otherwise $a_{i j}=a_{j i}=0, i \neq j$. The diagonal elements of matrix $A$ satisfy

$$
a_{i i}=-\sum_{j=1, j \neq i}^{N} a_{i j}=-\sum_{j=1, j \neq i}^{N} a_{j i} .
$$

Definition 1. In the dynamic network (1), the network is said to be consecutively synchronized for any initial conditions [29] if

$$
\lim _{t \longrightarrow \infty}\left\|x_{i}(t)-x_{j}(t)\right\|=0, \quad i, j=1,2, \ldots, N .
$$

The coupling matrix $A$ determined by the complex network structure reflects the synchronous state or consistent attribute of the network. Inspired by the nature of the coupling matrix, this paper constructs a consistent matrix with the abovementioned properties to characterize the synchronization or consistency of a complex network. When the system is in an abnormal state due to faults, the consistency will be destroyed, and the resulting abnormality will also be reflected in the coupling matrix. So, with the location and size of the matrix element that changes, the fault can be detected and located.

\subsubsection{Data-Driven Consistent Matrix Construction.}

Figure 1 shows that multimotor synchronous control is a typical complex network system. Considering the control objectives, this paper pays more attention to judging the consistency of the complex network output. To evaluate the 


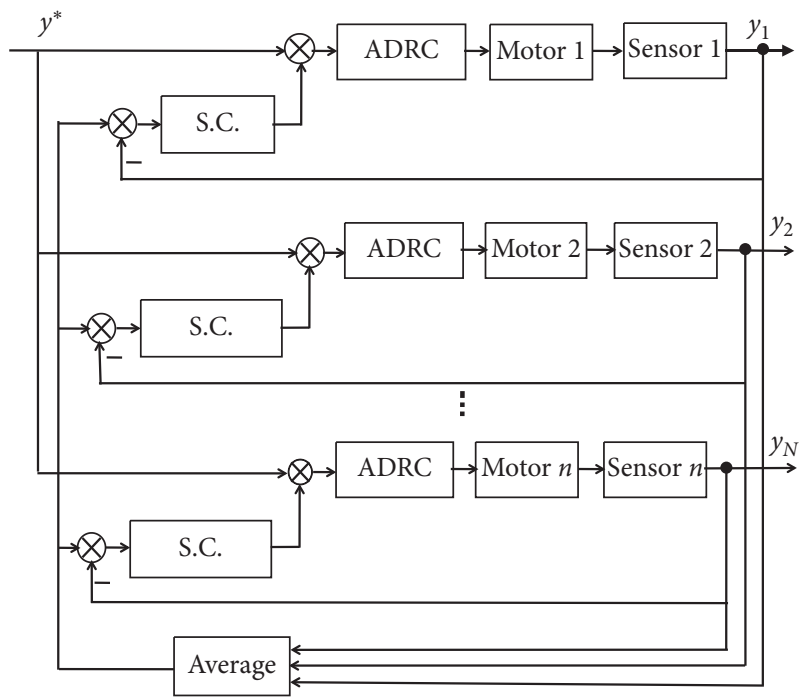

FIGURE 1: Deviation-coupling synchronization control structure.

consistency or similarity between different nodes in a complex network, a consistent matrix, which is derived from the coupling matrix, is introduced. The process of constructing the consistent matrix can be summarized as follows.

The output of the $N$ nodes of the multimotor synchronous complex network system shown in Figure 1 is measured by $N$ sensors and constitutes a set $Y=\left[y_{1}, y_{2}, \ldots, y_{N}\right]$. The consistency between $y_{i}$ and $y_{j}$ is represented by the coefficient $a_{i j}$. As $a_{i j}$ gets larger, $y_{j}$ and $y_{i}$ become more consistent. The consistency between two different nodes is the same, which means $a_{i j}=a_{j i}$. Thus, the matrix $A$ is a symmetric matrix. Constructing $A$ is very important to effectively distinguish the consistency between $y_{i}$ and $y_{j}$. Generally, it is set as an exponential function of the distance between $y_{i}$ and $y_{j}$. The construction form is given as follows:

$$
A(k)= \begin{cases}a_{i j}=e^{-\sqrt{\left(y_{i}(k)-y_{j}(k)\right)^{2}}}, & i \neq j, \\ a_{i i}=-\sum_{j=1, i \neq j}^{N} a_{i j}, & i=j,\end{cases}
$$

where $y_{i}(k)$ is the output data from the sensor on node $i$ at sampling moment $k, i, j=1,2, \ldots, N$. Matrix $A$ satisfies the conditions of the coupling matrix, that is, $a_{\mathrm{ij}}=a_{\mathrm{ji}}>0, i \neq j$, $\operatorname{rank}(A)=N-1$. It also has the same properties as the coupling matrix.

Remark 1 . The matrix is completely generated by the output data of the sensor, so it can be considered as data-driven based. At the same time, the structure of $A$ reflects the distance relationship between two different sensor outputs. Therefore, it has the characteristics of a model. Moreover, the design of $A$ can be considered as a combination of datadriven and model-based methods, which has the advantages of convenient data acquisition and reflecting the mechanism characteristics of the network model.
Remark 2. For the network synchronization, the abnormal or fault output of a certain node will be far away from the output of other normal nodes. It will decrease the consistency between them accordingly. This change can be directly reflected from the corresponding position in the matrix, which is the basis for follow-up fault diagnosis studies.

\subsection{Sensor Fault Diagnosis Based on Consistent Matrix}

3.2.1. Consistent Matrix Analysis under Normal/Fault Condition of the Sensor. Based on the design of the consistent matrix described above, for a multimotor synchronous control system shown in Figure 1, the following assumptions are made.

Assumption 1. There are $N$ motors to be synchronized, and the output of each subsystem can be regarded as a node of a complex network.

Assumption 2. The system has good synchronous control performance under normal conditions. The system reaches steady state and has good synchronization accuracy, which means that $y_{i} \approx y_{j}$.

Assumption 3. When two or more sensors fail at the same time, the sizes or magnitudes of faults are different.

Under the previous assumptions, we can obtain $a_{i j} \approx 1$ and $a_{i i} \approx-(N-1)$. The eigenvalues of $A$ satisfy $\lambda_{1}=\lambda_{2}=$ $\cdots=\lambda_{N-1}=-N$ and $\lambda_{N}=0$. When a sensor (taking the $i$ th sensor as an example) fails, its output will inevitably deviate from other normal sensor outputs. The elements in the $i$ th row and the $i$ th column of $A$ will show $a_{i *}<1, a_{* i}<1$ (except for the diagonal), and with the magnitude of the fault increases, $a_{i *} \ll 1, a_{* i} \ll 1$. The eigenvalues of matrix $A$ will also change accordingly.

\subsubsection{Fault Detection and Location Based on Consistent} Matrix Judgement. From the analysis above on different characteristics of the consistent matrix $A$ before and after the fault, fault detection can be performed by judging the element size of $A$. Generally, three types of fault detection methods can be selected:

(1) $A(k)$ is compared with $A(k-l)$. Taking the length of the data as $l$, generate $A(k-1), \ldots, A(k-l)$ and compare $A(k)$ with the elements at the corresponding position of $A(k-l)$. When a row or column of elements in the deviation matrix exceeds the predefined threshold, the fault can be detected and the fault sensor can be located according to the row or column position of the element exceeding the threshold.

(2) Elements in $A(k)$ are compared with 1 . With the analysis above, when the sensor is normal, $a_{i j} \approx 1$. When the $i$ th sensor fails, the $i$ th row and the $i$ th column elements in $A(k)$ appear to be much smaller than $1, a_{i *}<1, a_{* i}<1$. The fault sensor can be identified and located accordingly. 
(3) Using the change in $A(k)$ eigenvalues. The eigenvalues of the consistent matrix are also changed when a fault occurs. The normal eigenvalues are approximately $-N$ and 0 . After the fault occurs, the matrix $A(k)$ still satisfies the dissipation characteristics, although $\operatorname{rank}(A)=N-1$, except for the eigenvalue 0 , the distribution of other eigenvalues will change instead.

For the abovementioned three methods, from the perspective of detection speed and reliability, there are different characteristics. Method (1) uses $l$ sample data to test. With the sample number increasing, the reliability of the detection is improved, while a certain delay will occur. Therefore, from real-time and reliability consideration, $l$ should not be too large. Method (2) has fast diagnostic speed and good real-time performance, but the anti-interference performance is poor, possibly leading to false alarms. Method (3) is essentially the same as Method (2). They both are based on $A(k)^{\prime} s$ own elements or eigenvalues. However, the limitation of Method (3) lies in that it cannot locate the fault.

Combining the characteristics analysis of the abovementioned three methods, to improve the efficiency and reliability of the diagnosis, it is a good idea to combine Methods (1) and (2). When the diagnosis program is implemented, Method (2) is mainly used. After the fault is judged, the $A(k)$ comparison is made with the latter steps in combination. That is, Method (1) is used for further confirmation. Diagnosis speed, reliability, and computing resources can be simultaneously ensured.
It is worth mentioning that the diagnostic method above is also applicable to multiple fault situations. It means that when multiple sensors fail simultaneously, multirow and multicolumn element values will satisfy $a_{i j}<1$ at the corresponding positions in the matrix.

For example, when $M$ sensors fault simultaneously in a multimotor synchronous control system composed of $N$ Motors $(M<N)$ under Assumption 3, there will be $C_{N-M}^{2}=$ $(N-M)(N-M-1) / 2$ element values satisfying $a_{i j}=1$, while other $M(2 N-M-1) / 2$ elements will satisfy $a_{i j}<1$, except for diagonal elements. The diagnostic process is similar to the single fault scenario.

However, as the number of faulty sensors increases, the types of faults diversify and the number of elements to be judged will increase greatly. Accordingly, the diagnosis time is prolonged. At the same time, the distances between two different sensors, especially between the faulty sensors, are various. All of these will decrease the overall diagnostic reliability of the method to some extent, especially when the detection threshold is set to a certain fixed value.

\subsubsection{Fault Estimation Based on Eigenvalues of the Consistent} Matrix. In addition to detecting and locating the fault, the fault size can be estimated by further studying the performance of matrix $A$. The specific process is as follows.

When the $i$ th sensor fails, the consistent matrix $A$ can be approximated as (ignoring the noise and synchronization error)

$$
A^{f} \approx\left[\begin{array}{cccccc}
-(N-2+\beta) & 1 & \cdots & \beta & \cdots & 1 \\
1 & -(N-2+\beta) & \cdots & \beta & \cdots & 1 \\
\vdots & \vdots & \vdots & \vdots & \vdots & \vdots \\
\beta & \beta & \cdots & -(N-1) \beta & \cdots & \beta \\
\vdots & \vdots & \vdots & \vdots & \vdots & \vdots \\
1 & 1 & \cdots & \beta & \cdots & -(N-2+\beta)
\end{array}\right],
$$

where $\beta$ is the consistent coefficient between the fault sensor and other normal sensors, $\beta<1$. To estimate the fault size, the abovementioned matrix can be transformed into the following form:

$$
A^{f^{*}} \approx\left[\begin{array}{cccccc}
-(N-2+\beta) & 1 & \cdots & \beta & \cdots & 1 \\
1 & -(N-1) & \cdots & 1 & \cdots & 1 \\
\vdots & \vdots & \vdots & \vdots & \vdots & \vdots \\
\beta & 1 & \cdots & -(N-2+\beta) & \cdots & 1 \\
\vdots & \vdots & \vdots & \vdots & \vdots & \vdots \\
1 & 1 & \cdots & 1 & \cdots & -(N-1)
\end{array}\right] .
$$


That is, except for $a_{1 i}=a_{i 1}=\beta$, the diagonal elements remain unchanged from the original rule and the elements in other positions are all set to 1 . It is easy to obtain the eigenvalues of $A^{f^{*}}$, which are $\lambda_{1}=\cdots=\lambda_{N-2}=-N$, $\lambda_{N-1}=-(N-2+2 \beta)$, and $\lambda_{N}=0$. Compared with the matrix $A$ in normal situations, the only changed eigenvalue of $A^{f^{*}}$ is a fault-related quantity, i.e., $\lambda_{N-1}=-(N-2+2 \beta)$. Therefore, the fault size can be estimated as

$$
\begin{aligned}
\beta^{*} & =-\frac{N-2+\lambda_{N-1}^{*},}{2}, \\
\left|y_{1}-y_{i}\right| & =\ln \frac{1}{\beta^{*} .}
\end{aligned}
$$

Considering that $A^{f}$ is a singular matrix, to obtain $A^{f^{*}}$, a generalized inverse of $A^{f}$ can be used to obtain a transform matrix, $P=A^{f^{*}} \cdot\left(A^{f}\right)^{+}$.

Remark 3. Compared with some traditional model-based methods, which have a complicated process of modelling, observer designing, parameter optimization, etc., the proposed method can realize the detection, location, and estimation of sensor faults only by judging the elements and eigenvalues of the consistent matrix. It is completely driven by the output data of the sensor and applicable for single and multiple fault situations. Therefore, the proposed method has some significant advantages, such as simple calculation, convenient implementation, and clear physical meaning.

3.3. Fault Tolerance Based on Improved Weighted Mean Feedback. After the fault is diagnosed, timely and effective isolation and fault tolerance of the faulty sensor are essential to ensure the safe and stable operation of the system. The signal collected by the sensor is used as the input signal of the mean feedback and the self-feedback. Therefore, after detecting the fault, the two signals should be isolated and reconstructed to ensure the security of the system. For the mean feedback part, it is modified from the weighted mean form as follows:

$$
\bar{y}=\frac{1}{\sum_{i=1}^{N} \alpha_{i}} \sum_{i=1}^{N} \alpha_{i} y_{i}
$$

where $y_{i}$ is the output signal of each sensor and $\alpha_{i}$ can be defined as the reliability coefficient of the $i$ th sensor. Based on the abovementioned fault diagnosis result, when the sensor is normal, that is, completely reliable, $\alpha_{i}=1$, while when the sensor is faulty, $\alpha_{i}=0$, as seen in the following equation:

$$
\alpha_{i}= \begin{cases}1, & \text { Normal }, \\ 0, & \text { Fault. }\end{cases}
$$

When the sensor is normal, the feedback signal is the traditional average form $1 / N \sum_{i=1}^{N} y_{i}$, and when the sensor is faulty because the reliability coefficient is introduced, $\alpha_{i}=0$, the corresponding sensor signal is cut off and the fault sensor is automatically isolated. As a result, fault sensor isolation and system fault tolerance can be realized. It does not affect the generation of the mean signal, nor does it change the topology of the original network.

For the self-feedback part, the mean signal $\bar{y}$ is used to replace the faulty sensor output $y_{i}$. In this situation, the system output keeps the current value unchanged.

Based on the analysis above, for the sensor failure problem in a multimotor synchronous control system, the synchronous control with improved deviation-coupling structure of fault diagnosis and fault tolerance function is shown in Figure 2.

The outputs of the FDD and FTC module are the modified outputs $\tilde{y}_{1}, \tilde{y}_{2}, \ldots, \tilde{y}_{N}$ of sensors 1 to $N$, the fault indicator $\gamma$, and the mean feedback $\bar{y}$, respectively. When the sensor is normal, $\tilde{y}_{i}=y_{i}$, and when the sensor is faulty, the corresponding output is $\tilde{y}_{i}=\bar{y}$.

Remark 4. The original mean value is improved by introducing the reliability coefficient, and the modified mean feedback design is performed accordingly. The proposed method does not affect the mean feedback output or the system topology and can implement faulttolerant control to assure the reliability and safety of the system.

3.4. Sensor Fault Detection, Isolation, and Tolerance Steps. Based on the abovementioned analysis, for a single sensor fault, the fault diagnosis, isolation, and fault tolerance processes are as follows:

Step 1. Initialization: set the sampling period $T_{s}$ and the diagnostic threshold th of the system operation; label the sensors in the multimotor synchronous system from 1 to $N$; and let $\alpha_{i}=1, i=1,2, \ldots, N$, and the fault indication output $\gamma=0$.

Step 2. Generating matrix $A(k)$ : the consistent matrix $A(k)$ is generated according to (4) by the output of each subsystem.

Step 3. Fault diagnosis: judging if each element of $A(k)$ satisfies $a_{\mathrm{ij}}<$ th. If yes, the fault occurs and the algorithm proceeds to Step 4; otherwise, it returns to Step 2 to generate the next moment matrix $A(k+1)$ and judges again.

Step 4. Fault tolerance: according to the diagnosis result of Step 3, if the $j$ th sensor fails, set $\alpha_{j}=0$, calculate $\bar{y}$ according to (8), and set $y_{j}=\bar{y}$ and $\gamma=j$.

It should be noted that to improve the efficiency of diagnosis, it only needs to judge the first row or the first column of matrix $A(k)$, not all the elements. The result of the judgment is nothing more than the following three cases. (1) If all the values of the first row or the first column satisfy $a_{1 i}<$ th or $a_{i 1}<$ th, the No.1 sensor fails and the fault indication output is $\gamma=1$; (2) if only one of the values satisfies $a_{1 i}<$ th, the No. $i$ sensor fails, the fault indication output is 


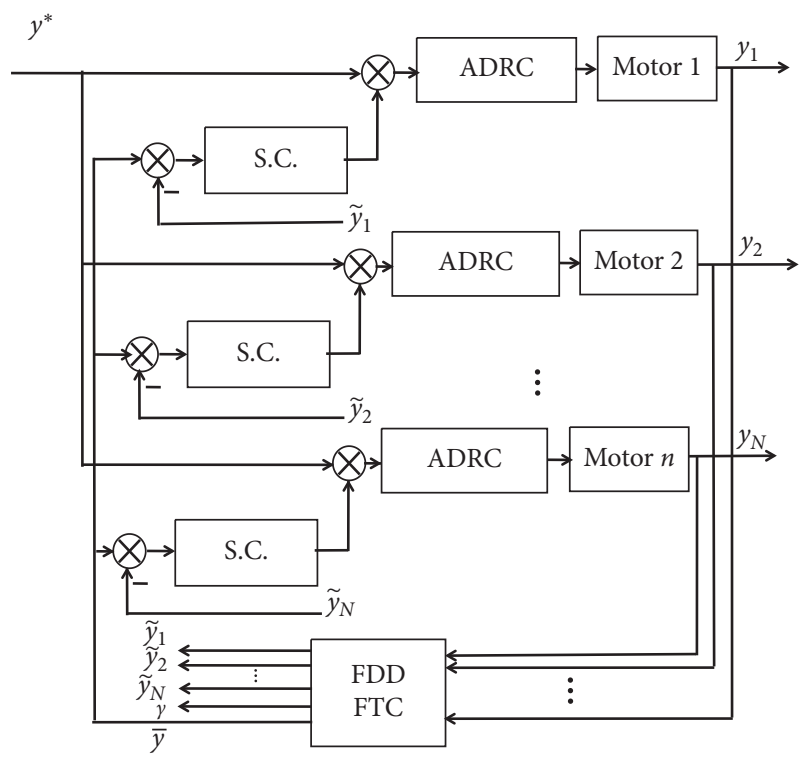

FIGURE 2: Improved structure with FDD and FTC functions.

$\gamma=i$; and (3) if all do not satisfy $a_{1 i}<$ th, no fault occurs, and the fault indication remains $\gamma=0$.

\section{Simulation Results and Analysis}

To verify the effectiveness of the fault diagnosis and faulttolerant method proposed above, a simulation is carried out for both a single sensor fault and multisensor simultaneous fault in the MATLAB/Simulink environment based on the control structure shown in Figure 2.

4.1. Single Sensor Fault Simulation. For a single sensor fault situation, the synchronous control of three permanent magnet synchronous motors (PMSM) is taken as an example. To verify the advantages of the proposed method, under different speed inputs, the sensors in subsystems 1-3 have three different types of faults such as constant deviation, stuck, and constant gain at different times. The PMSM models are shown in (10)-(13), and the parameters of each motor are given in Table 1 . The simulation time is $35 \mathrm{~s}$, the simulation step size is $T_{s}=0.001 \mathrm{~s}$, and threshold th $=0.1$. The simulation results are shown in Figures 3-6:

$$
\begin{aligned}
& \dot{i_{d}}=\frac{1}{L_{d}}\left(u_{d}-R_{s} i_{d}+\omega L_{q} i_{q}\right), \\
& \dot{i_{q}}=\frac{1}{L_{q}}\left(u_{q}-R_{s} i_{q}-\omega L_{d} i_{d}-\omega \phi_{f}\right), \\
& \dot{\omega}=\frac{1}{J}\left(P_{n} T_{e}-P_{n} T_{l}-B \omega\right), \\
& T_{e}=1.5 P_{n} \phi_{f} i_{q} .
\end{aligned}
$$

First, to reflect the impact of the fault, three types of fault shown in Table 2 are first applied to the No. 1 sensor only. Figure 7 shows the result of no action taken after the fault
TABLE 1: Three-motor mechanical parameters.

\begin{tabular}{lcccc}
\hline Variable & Symbol & Motor 1 & Motor 2 & Motor 3 \\
\hline Rated power $(\mathrm{kW})$ & $P$ & 1.5 & 1.5 & 1.5 \\
Rated speed $(\mathrm{rpm})$ & $N$ & 1500 & 1500 & 1500 \\
$\begin{array}{l}\text { Rated torque }(\mathrm{N} \cdot \mathrm{m}) \\
\text { D-axis self-inductance }\end{array}$ & $T$ & 3 & 3 & 3 \\
$(\mathrm{mH})$ & $L_{d}$ & 3.5 & 4.5 & 3 \\
$\begin{array}{l}\mathrm{Q}-\mathrm{axis} \text { self-inductance } \\
(\mathrm{mH})\end{array}$ & $L_{q}$ & 3.5 & 4.5 & 3 \\
$\begin{array}{l}\text { Stator resistance }(\Omega) \\
\text { Moment of inertia }\end{array}$ & $R_{s}$ & 0.94 & 0.89 & 1.32 \\
$\left(\mathrm{~kg} \cdot \mathrm{m}^{2}\right)$ & $J$ & 0.01 & 0.007 & 0.005 \\
Number of pole pairs & $p$ & 2 & 2 & 2 \\
\hline
\end{tabular}

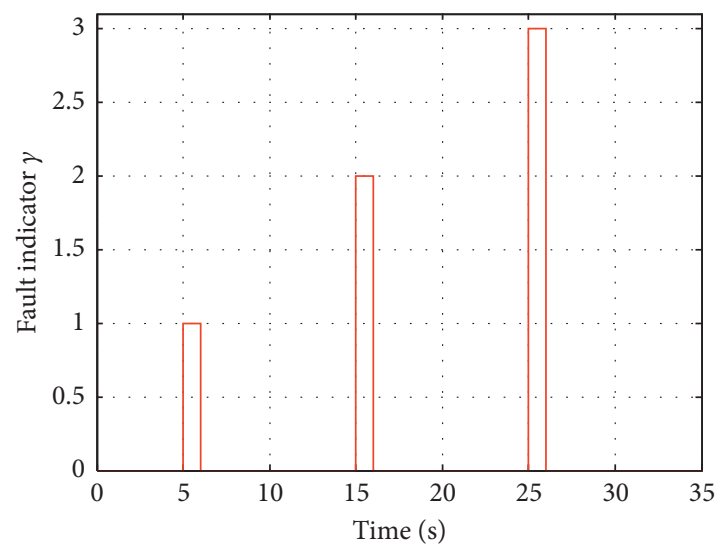

FIgURE 3: Fault indicator output of the method.

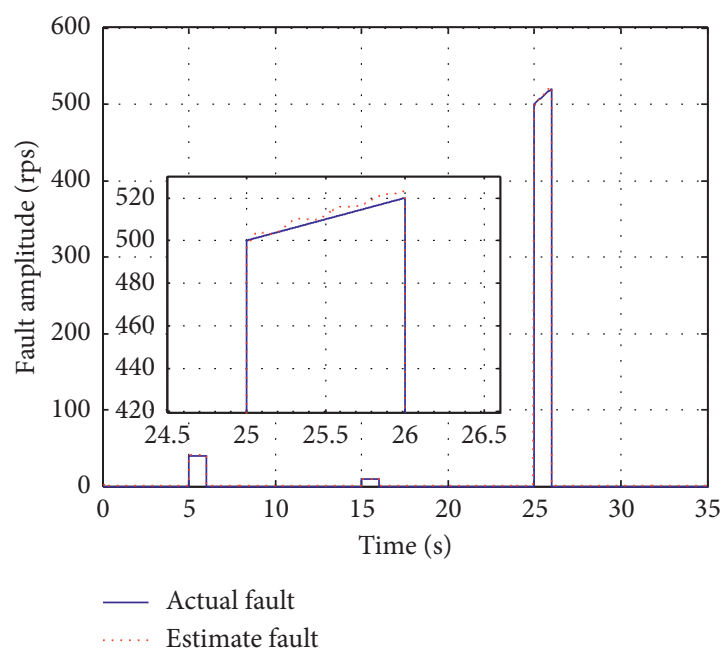

FIGURE 4: Fault estimation of the method in this paper.

occurs. A fault of the No. 1 sensor seriously affects the outputs of subsystems 2 and 3 at this moment. Clearly, the whole system cannot be synchronized for a period from the occurrence to the end of a fault.

Second, to verify the effect of the proposed fault diagnosis method, different types of faults, as shown in Table 2, are applied to three sensors at different times. Figure 3 shows the fault indicator results. When different types of sensor 


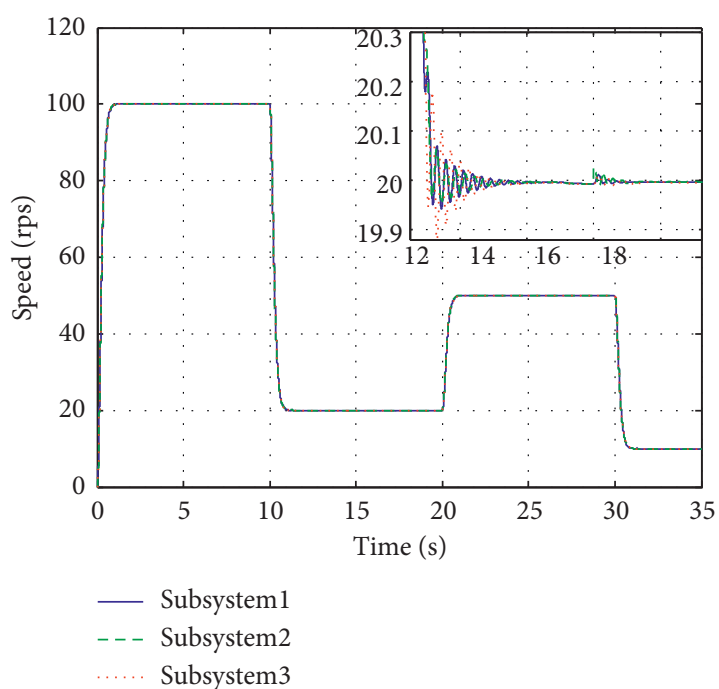

Figure 5: Tolerance synchronous output after the fault.

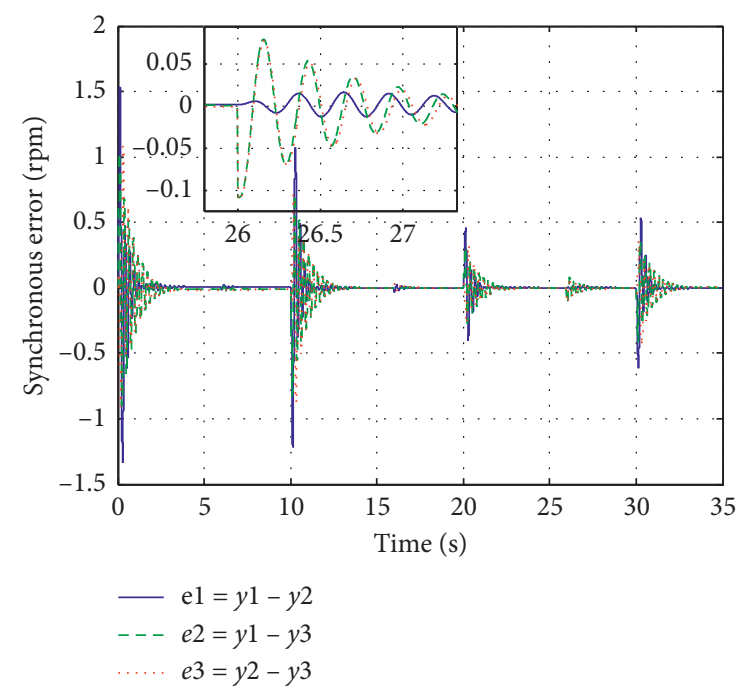

Figure 6: Tolerance synchronous output error.

TABLE 2: Sensor failure information.

\begin{tabular}{lccc}
\hline Sensor & Fault type & Mathematical description & Time \\
\hline 1 & Constant deviation & $\tilde{y}(k)=y(k)+c_{1}$ & $5-6 \mathrm{~s}$ \\
2 & Stuck & $\tilde{y}(k)=c_{2}$ & $15-16 \mathrm{~s}$ \\
3 & Constant gain & $\tilde{y}(k)=c_{3} y(k)$ & $25-26 \mathrm{~s}$ \\
\hline
\end{tabular}

fault occur at 5, 15, and $25 \mathrm{~s}$, the fault indicator output is $\gamma=1,2$, and 3 , respectively. It is clear to see that the proposed method can detect and locate the fault quickly and accurately. Figure 4 shows the results of fault estimation. The maximum estimation error shown in the detail figure is only about $3 \mathrm{rad} / \mathrm{s}$. The estimation is accurate.

Third, with the abovementioned diagnosis results, the improved weighted mean feedback based on the reliability coefficient was used to isolate the faulty sensor and achieve fault-tolerant control. The results are shown in Figure 5 and 6.

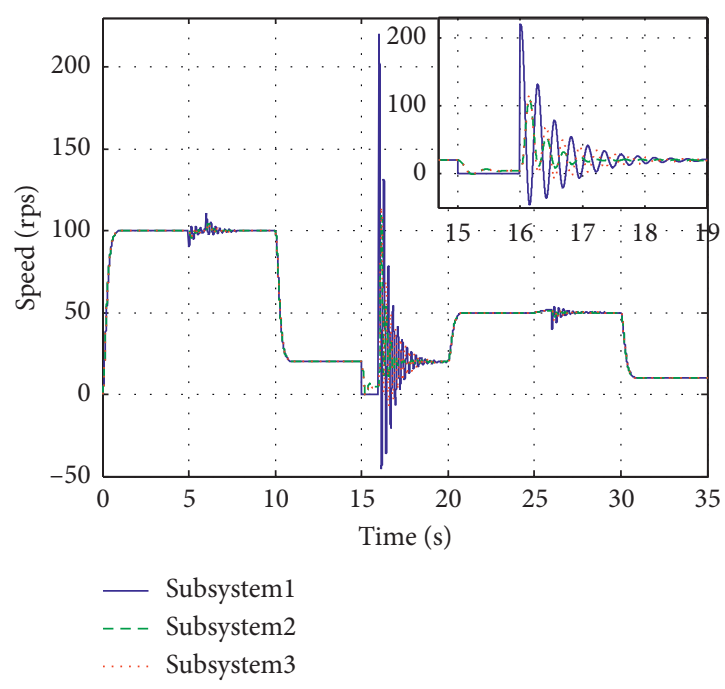

FIgURE 7: No FDD synchronous output after failure.

It can be seen from Figure 5 that the system still maintains a satisfactory synchronization accuracy after the fault occurs, in addition to the slight fluctuations caused by the adjustment of the controller in a short time. Furthermore, from the synchronous output error of Figure 6, one can see that the error caused by the fault tolerance is much smaller than the error of control when the desired rotational speed is changed. The maximum tolerance error is about $0.1 \mathrm{rad} / \mathrm{s}$.

4.2. Multisensor Fault Simulation. As mentioned above, the proposed method is also suitable for multifault situation. So, another simulated synchronous system composed of four motors is presented, considering the simultaneous failure of two sensors. The parameters of the four motors are shown in Table 3. The simulation time is $35 \mathrm{~s}$, th $=0.1$, and the simulation step size $T_{s}=0.001 \mathrm{~s}$.

For the synchronous control system consisting of four motors, when two sensors fail simultaneously, there are six combinations. Three combinations of them can be taken into consideration. Three types of sensor failures (constant gain, stuck, and constant deviation) are considered. The fault information is shown in Table 4.

Figure 8 shows the results of the system synchronization output after 2 sensors fail simultaneously without taking any measures. Similar to the single failure scenario, the synchronization performance of the system is degraded for a period of time. Figures 9-11 show the results of FDD and FTC using the proposed method.

It can be seen from the time indicated by the fault shown in Figure 9 that the method can detect the fault quickly and exactly in 5-6, 15-16, and 25-26 s. Fault indicator amplitudes 12, 34, and 24 indicated that No. 1-No. 2, No. 3-No. 4, and No. 2-No. 4 failed simultaneously, respectively. The fault location is also satisfied for multisensor faults.

After the fault is diagnosed, Figure 10 shows the faulttolerant results using the improved weighted mean feedback. When two sensors fail at the same time, only the outputs of 
TABLE 3: 4 motor mechanical parameters.

\begin{tabular}{lcccc}
\hline Variable & Motor 1 & Motor 2 & Motor 3 & Motor 4 \\
\hline Rated speed $(\mathrm{rpm})$ & 1500 & 1500 & 1500 & 4.5 \\
Rated torque $(\mathrm{N} \cdot \mathrm{m})$ & 3 & 3.3 & 4.1 & 4 \\
D-axis self-inductance $(\mathrm{mH})$ & 3.5 & 3.7 & 4.0 & 3.8 \\
Q-axis self-inductance $(\mathrm{mH})$ & 3.2 & 3.6 & 1.32 & 3.6 \\
Stator resistance $(\Omega)$ & 0.9 & 0.94 & 0.005 & 1 \\
Moment of inertia $\left(\mathrm{kg} \cdot \mathrm{m}^{2}\right)$ & 0.01 & 0.007 & 2 \\
Number of pole pairs & 2 & 2 & 0.006 \\
\hline
\end{tabular}

TABle 4: Sensor fault information.

\begin{tabular}{|c|c|c|c|c|}
\hline Sensor & Fault type & Mathematical description & Fault amplitude & Time $(\mathrm{s})$ \\
\hline No. 1 , No. 2 & Constant deviation & $\tilde{y}(k)=y(k)+c_{1}$ & $c_{1}=-40, c_{1}^{\prime}=-20$ & $5-6$ \\
\hline No. 3 , No. 4 & Stuck & $\tilde{y}(k)=c_{2}$ & $c_{2}=22, c_{2}^{\prime}=18$ & $15-16$ \\
\hline No. 2 , No. 4 & Constant gain & $\tilde{y}(k)=c_{3} y(k)$ & $c_{3}=100, c_{3}^{\prime}=200$ & $25-26$ \\
\hline
\end{tabular}

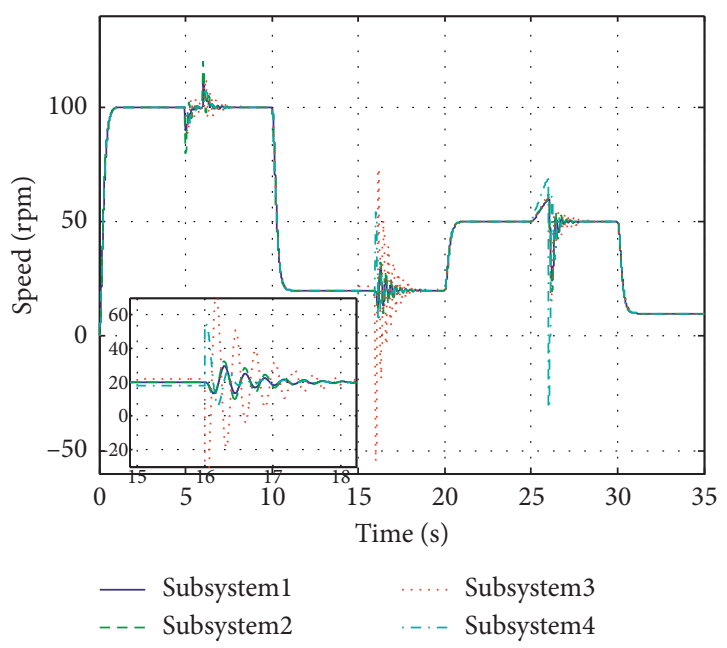

FIGURE 8: Synchronous output without taking any action when two sensors fail simultaneously.

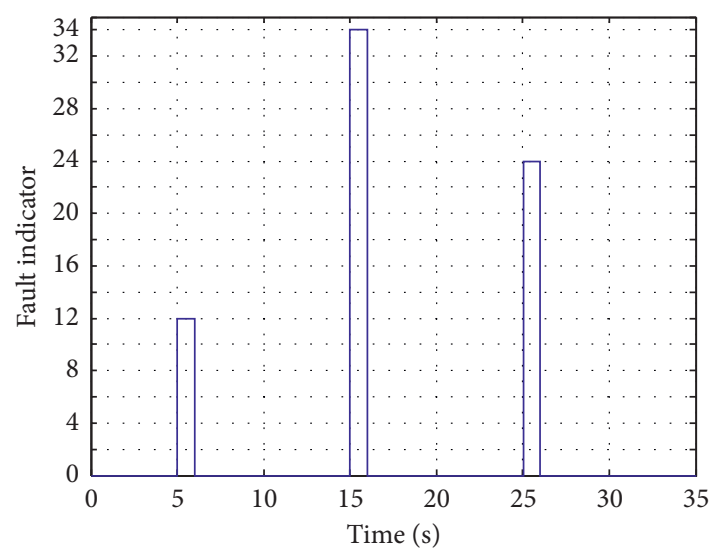

Figure 9: Fault indication output when two sensors fail simultaneously.

the remaining two normal sensors are used, and a satisfactory synchronization performance is achieved. Furthermore, from Figure 11, the synchronization error at $t=25 \mathrm{~s}$ is

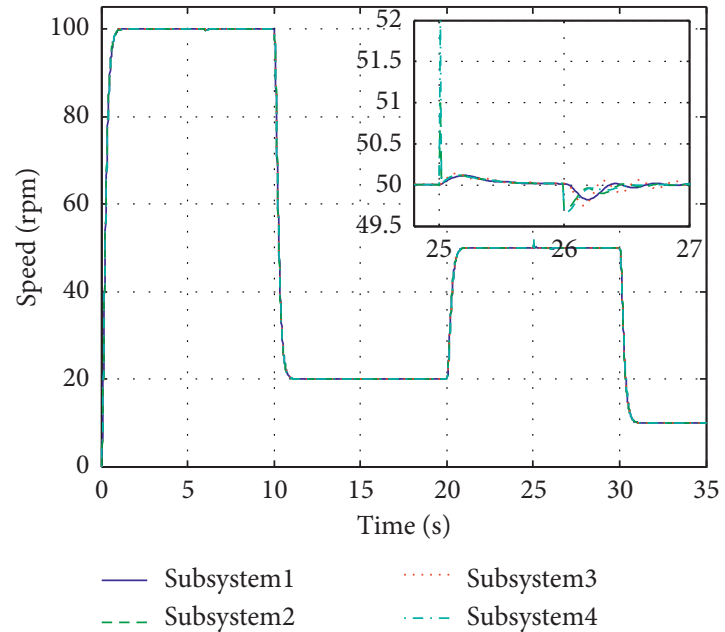

FIgURE 10: Synchronous output of FTC.

relatively large (about $2 \mathrm{rad} / \mathrm{s}$ ) due to the slow change characteristics of constant gain fault. The synchronization error of tolerance is far less than the control error of the expected speed change. The fault tolerance is satisfactory overall.

It should be pointed out that, when two sensors have different amplitude faults, the distances between the faulty sensor and a normal sensor, as well as between two faulty sensors, become complicated and diverse. It is difficult to estimate the fault size. In addition, considering the existence of the synchronization error and the randomness of the distance between different sensors outputs, the threshold selection is more sensitive.

\section{Experimental Results and Analysis}

To test the engineering applicability of the proposed method, a multimotor synchronous control experimental platform composed of 4 motors is established. The hardware mainly includes ABB's AC500-eCo PLC; input and output modules DX561, DC562, and AX561; ACS355 frequency 


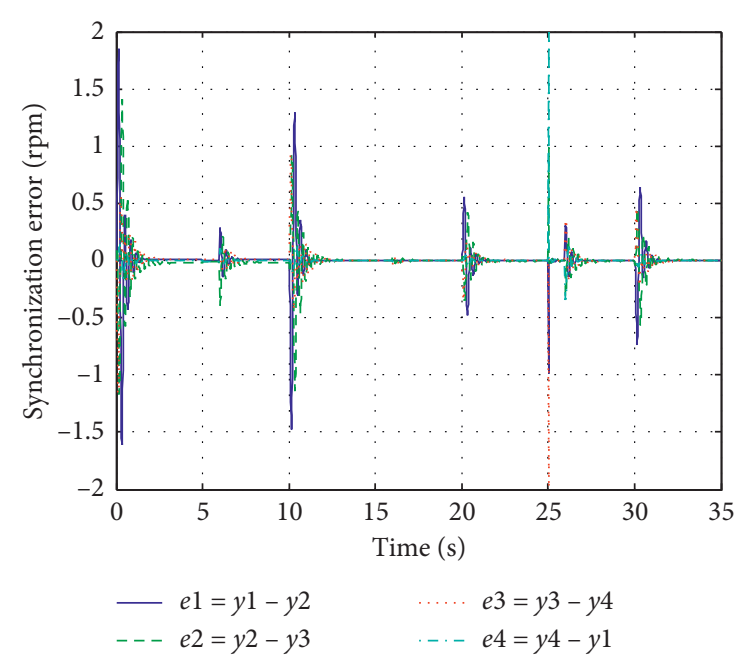

Figure 11: Synchronization error of FTC.

TABLe 5: Experimental motor mechanical parameters.

\begin{tabular}{lcc}
\hline Variable & Symbol & Motor \\
\hline Power (kW) & $P$ & 0.4 \\
Rated voltage (V) & $U$ & 220 \\
Rated current (A) & $I$ & 1.8 \\
Rated speed (rpm) & $N$ & 3000 \\
Rated torque (N.m) & $T$ & 1.2 \\
Number of pole pairs & $p$ & 1 \\
\hline
\end{tabular}

converter and permanent magnet synchronous motor (see Table 5 for parameter information); and 360 line photoelectric encoder. The software mainly includes Control Builder Plus (referred to as CBP, integrated with CoDeSys), OPC configurator, and MATLAB/Simulink. Because the AC500-eCo PLC contains only two high-speed counting channels, the four motors are controlled by two PLCs through four frequency converters. The established experimental platform is shown in Figure 12.

Setting sensor No. 2-4 to have three kinds of fault, constant deviation, constant gain, and stuck, the corresponding fault occurrence and duration time is $1-1.5 \mathrm{~s}$, $2.5-3 \mathrm{~s}$, and $4-4.5 \mathrm{~s}$, respectively. The expected speed is $2000 \mathrm{rpm}$. The results of fault diagnosis and fault tolerance are shown in Figures 13 and 14.

The fault diagnosis is timely and reliable. The design of the improved weighted average feedback mechanism based on the diagnosis results realized the fault-tolerant control. The synchronous precision of the system after fault tolerance is satisfactory in the semiphysical experiment. The effectiveness of the method is verified once again in the platform. It also demonstrates that the fault diagnosis method based on complex network consistent matrix and the tolerance design of improved weighted mean feedback have good engineering applicability.

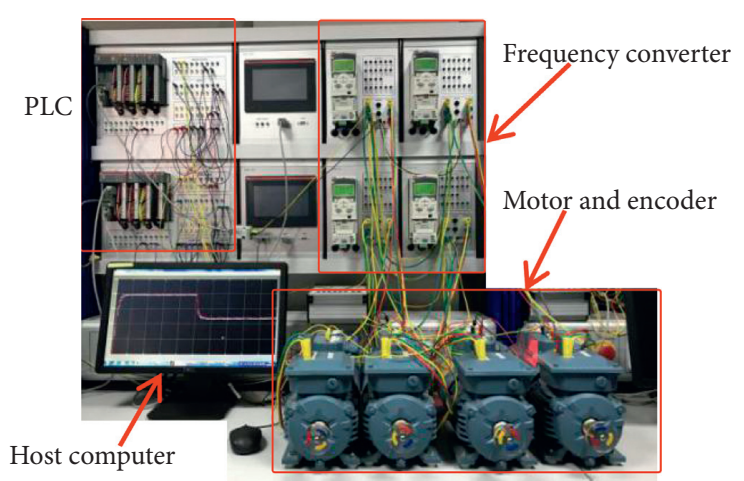

FIGURE 12: Semiphysical experiment platform.

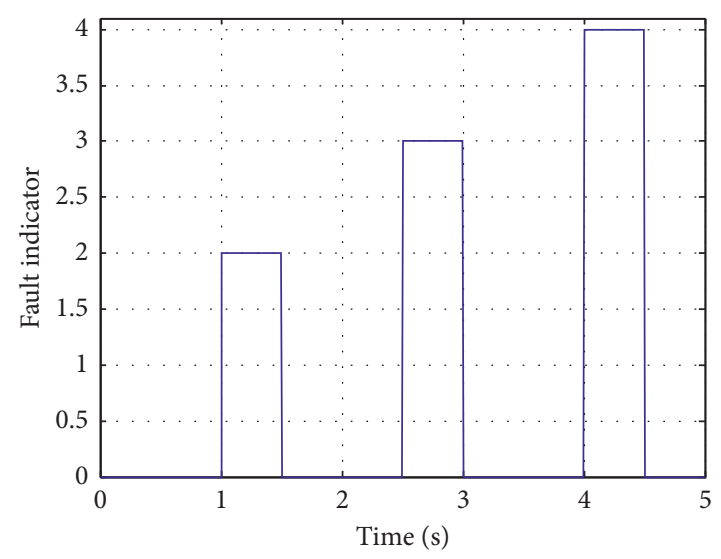

FIGURE 13: Fault indication output.

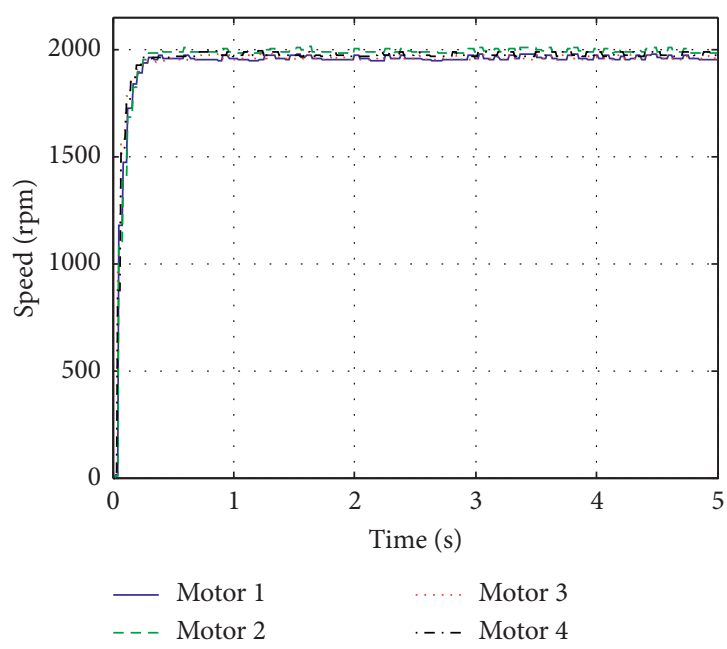

FigUre 14: Synchronous output after fault tolerance.

\section{Conclusion}

A fault diagnosis and fault-tolerant control method based on complex network synchronization has been presented to 
mitigate sensor fault problems in multimotor synchronous control. Based on the concept of distance, inspired by the idea of the coupling matrix in complex network synchronization, a consistent matrix is devised, which can reflect the similarity of different sensor output data. From the online correlation of elements and features in the matrix, the sensor fault can be detected, located, and estimated by the element value judgments in the matrix. Based on the fault diagnosis information, a fault-tolerant mechanism has been designed by introducing an improved weighted mean feedback to achieve effective isolation of the faulty sensor. Compared with the existing theories and techniques, the proposed method has several advantages, such as simple principles, small calculation amount, no need to change the topology structure of the original network, easy engineering realization, and suitability for single and multiple failure.

From simulations and experiments, in order to implement satisfactory synchronous control, by considering load interference, noise, and uncertainty, a strong robustness control algorithm is necessary when the sensor is normal. In the meantime, the proposed method can be superimposed on the system as a further synchronous output guarantee. The reason is that, due to interference and other factors, when the synchronization error is large at a certain time, the method has the function of automatically rejecting the deviation from the larger output, and adopting other closer output averages as the feedback function, which makes the mean feedback closer to the true value. Therefore, this method can achieve better performance than conventional mean feedback, that is, it can realize fault tolerance when there is a fault and improve synchronization accuracy when there is no fault.

In our future work, the focus will be on the robust and adaptive threshold selection study for multisensor fault situation.

\section{Data Availability}

The simulation and experimental data used to support the findings of this study are available from the corresponding author upon request.

\section{Conflicts of Interest}

The authors declare no conflicts of interest.

\section{Acknowledgments}

This work was supported in part by the National Natural Science Foundation of China (Grant nos. 61763027 and 61873116).

\section{References}

[1] I. X. Bogiatzidis, A. N. Safacas, E. D. Mitronikas, and G. A. Christopoulos, "A novel control strategy applicable for a dual AC drive with common mechanical load," IEEE Transactions on Industry Applications, vol. 48, no. 6, pp. 2022-2036, 2012.
[2] M. A. Valenzuela and R. D. Lorenz, "Electronic line-shafting control for paper machine drives," IEEE Transactions on Industry Applications, vol. 37, no. 1, pp. 158-164, 2001.

[3] D. Xiao, X. Li, and K. He, "Power balance of starting process for pipe belt conveyor based on master-slave control," IEEE Access, vol. 6, pp. 16924-16931, 2018.

[4] H.-H. Lian, S.-P. Xiao, Z. Wang, X.-H. Zhang, and H.-Q. Xiao, "Further results on sampled-data synchronization control for chaotic neural networks with actuator saturation," Neurocomputing, vol. 346, pp. 30-37, 2019.

[5] X. Chu, X. Nian, M. Sun, H. Wang, and H. Xiong, "Robust observer design for multi-motor web-winding system," Journal of the Franklin Institute, vol. 355, no. 12, pp. 52175239, 2018.

[6] R. Shu, J. Wei, D. Qin, T. C. Lim, and A. Zhang, "Global sensitivity analysis and dynamic optimization of multi-motor driving transmission system," Structural and Multidisciplinary Optimization, vol. 58, no. 2, pp. 797-816, 2018.

[7] M. Wang, X. Ren, and Q. Chen, "Robust tracking and distributed synchronization control of a multi-motor servomechanism with $\mathrm{H}$-infinity performance," ISA Transactions, vol. 72, pp. 147-160, 2018.

[8] K. L. Shi, T. F. Chan, Y. K. Wong, and S. L. Ho, "Speed estimation of an induction motor drive using an optimized extended Kalman filter," IEEE Transactions on Industrial Electronics, vol. 49, no. 1, pp. 124-133, 2002.

[9] M. Bourogaoui, H. B. A. Sethom, and I. S. Belkhodja, "Speed/ position sensor fault tolerant control in adjustable speed drives-a review," ISA Transactions, vol. 64, pp. 269-284, 2016.

[10] H. J. Mao, W. Li, K. H. Wang et al., "Sensor fault-tolerant switch strategy study for multi-motor synchronous system based on ADRC," Journal of Shandong University, vol. 47, no. 5, pp. 64-70, 2017.

[11] T. A. Najafabadi, F. R. Salmasi, and P. Jabehdar-Maralani, "Detection and isolation of speed-, DC-link voltage-, and current-sensor faults based on an adaptive observer in induction-motor drives," IEEE Transactions on Industrial Electronics, vol. 58, no. 5, pp. 1662-1672, 2011.

[12] R. Marino, S. Scalzi, P. Tomei, and C. M. Verrelli, "Faulttolerant cruise control of electric vehicles with induction motors," Control Engineering Practice, vol. 21, no. 6, pp. 860-869, 2013.

[13] B. Cai, Y. Zhao, H. Liu, and M. Xie, "A data-driven fault diagnosis methodology in three-phase inverters for pmsm drive systems," IEEE Transactions on Power Electronics, vol. 32, no. 7, pp. 5590-5600, 2017.

[14] C. M. Verrelli, S. Bifaretti, E. Carfagna et al., "Speed sensor fault tolerant PMSM machines: from position-sensorless to sensorless control," IEEE Transactions on Industry Applications, vol. 55, no. 4, pp. 3946-3954, 2019.

[15] A. B. Youssef, S. K. El Khil, and I. S. Belkhodja, "Open-circuit fault diagnosis and voltage sensor fault-tolerant control of a single-phase pulsed width modulated rectifier," Mathematics and Computers in Simulation, vol. 131, pp. 234-252, 2017.

[16] X.-J. Li and G.-H. Yang, "Adaptive fault-tolerant synchronization control of a class of complex dynamical networks with general input distribution matrices and actuator faults," IEEE Transactions on Neural Networks and Learning Systems, vol. 28, no. 3, pp. 559-569, 2017.

[17] C. Chen, X. Zhou, Z. Li, Z. He, Z. Li, and X. Lin, "Novel complex network model and its application in identifying critical components of power grid," Physica A: Statistical Mechanics and Its Applications, vol. 512, pp. 316-329, 2018. 
[18] L. Ding, Q.-L. Han, and X.-M. Zhang, "Distributed secondary control for active power sharing and frequency regulation in islanded microgrids using an event-triggered communication mechanism," IEEE Transactions on Industrial Informatics, vol. 15, no. 7, pp. 3910-3922, 2019.

[19] C. Zhang, J. Wang, D. Zhang, and X. Shao, "Fault-tolerant adaptive finite-time attitude synchronization and tracking control for multi-spacecraft formation," Aerospace Science and Technology, vol. 73, pp. 197-209, 2018.

[20] M. Tomizuka, J. Hu, and K. T. Chiu, "Synchronization of two motion control axes under adaptive feed-forward control," ASME Journal of Dynamic Systems. Measurement and Control, vol. 114, pp. 3234-3245, 1992.

[21] F. Perez-Pinal, G. Caladeron, R. Alvarez et al., "Comparison of multi-motor synchronization techniques," in Proceedings of the 30th Annual Conference of the IEEE Industrial Electronics Society, pp. 1670-1675, Busan, Korea, November 2004.

[22] T. Shi, H. Liu, Q. Geng, and C. Xia, "Improved relative coupling control structure for multi-motor speed synchronous driving system," IET Electric Power Applications, vol. 10, no. 6, pp. 451-457, 2016.

[23] F.-J. Lin, P.-H. Chou, C.-S. Chen, and Y.-S. Lin, "DSP-based cross-coupled synchronous control for dual linear motors via intelligent complementary sliding mode control," IEEE Transactions on Industrial Electronics, vol. 59, no. 2, pp. 1061-1073, 2012.

[24] W. Limei and H. Fei, "Research on synchro drive technique of dual motor based on decoupling control and internal model control," in Proceedings of the 2009 Chinese Control and Decision Conference (CCDC), pp. 5334-5337, Guilin, China, June 2009.

[25] L. Zhou, J. She, X. Zhang et al., "Performance enhancement of repetitive-control systems and application to tracking control of chuck-workpiece systems," IEEE Transactions on Industrial Electronics, vol. 67, no. 5, pp. 4056-4065.

[26] L. Zhou, J. She, S. Zhou, and C. Li, "Compensation for statedependent nonlinearity in a modified repetitive control system," International Journal of Robust and Nonlinear Control, vol. 28, no. 1, pp. 213-226, 2018.

[27] B.-L. Zhang, Q.-L. Han, X. Zhang et al., "Sliding mode control with mixed current and delayed states for offshore steel jacket platforms," IEEE Transactions on Control Systems Technology, vol. 22, no. 5, pp. 1769-1783, 2014.

[28] J. Chen, J. A. Lu, and J. Zhou, "On the relationship between the synchronous state and the solution of an isolated node in a complex network," Acta Automatic Sinica, vol. 39, no. 12, pp. 2111-2120, 2013.

[29] L. Guo and X. M. Xu, Complex Network, Shanghai Science and Technology Education Publishing House, Shanghai, China, 2006. 